\title{
Validation of the Substance Abuse Screener in American Sign Language (SAS-ASL)
}

\author{
Debra Guthmann \\ California School for the Deaf, Fremont, California
}

Dennis Moore

Wright State University

\author{
Linda E. Lazowski \\ SASSI Institute, Springville, Indiana
}

Allen W. Heinemann

Northwestern University and Rehabilitation Institute of Chicago, Chicago, Illinois

\author{
Jared Embree \\ Wright State University
}

\begin{abstract}
Objective: The study objectives were to adapt and validate a substance use disorder (SUD) screening instrument in American Sign Language (ASL) to be used to identify those deaf individuals who have a high probability of having an SUD. The goal was to develop an accurate screening instrument that balanced sensitivity and specificity while imposing minimal response burden on respondents. Method: A sample of 198 deaf participants in behavioral health, family social service, and educational programs that provide specialized services for deaf individuals was interviewed to obtain clinical diagnoses for current (past 12 months) SUD according to criteria of the Diagnostic and Statistical Manual of Mental Disorders, 4th edition, and completed a 42-item version of the Substance Abuse Screener in American Sign Language (SAS-ASL). We used Rasch and discriminant function analyses to reduce the instrument to 28 items, then divided the sample into a development subsample, used to formulate a scoring routine, and a validation subsample to assess correspondence with clinical diagnoses. To provide validation data on the shortened SAS - ASL, an independent sample of 62 respondents was diagnosed and completed the screener. Results: The SAS-ASL instrument demonstrated good person reliability (.85), sensitivity (.90), and specificity (.84) in the primary validation sample, and $100 \%$ screening accuracy with 62 respondents in the second validation sample. Conclusion: The SAS-ASL provides a standardized SUD screening for the deaf population. The adaptability of the instrument to electronic administration lends itself to a continuum of technologically supported services for a high-risk population that is disenfranchised for most community-based behavioral health services.
\end{abstract}

Keywords: substance use disorders, deaf, alcohol and drug screening, American Sign Language

\section{Impact and Implications}

- Clinicians' need for an efficient and accurate means of identifying substance use disorders in deaf individuals is met with the Substance Abuse Screener in American Sign Language (SAS-ASL).

- The SAS-ASL can be used to identify deaf persons who need further evaluation for substance use disorders and to facilitate treatment planning.

- Greater awareness of substance use disorder treatment needs will help more deaf persons obtain the services they need for conditions which historically have not been identified.

\section{Introduction}

The need for an efficient and accurate means of identifying substance use disorders (SUDs) in deaf individuals has been largely unaddressed, owing in part to the lack of empirically validated screening tools for use in this population. Researchers have reported that the prevalence of substance abuse in the deaf community is at least equivalent to the hearing population (Lipton \& Goldstein, 1997; Austen \& Checinski, 2000; Moore \& McAweeney, 2006/2007). According to the most recent U.S. national survey data on SUDs, $8.9 \%$
Debra Guthmann, California School for the Deaf, Fremont, California; Linda E. Lazowski, SASSI Institute, Springville, Indiana; Dennis Moore and Jared Embree, Department of Community Health, Boonshoft School of Medicine, Wright State University; Allen W. Heinemann, Center for Rehabilitation Outcomes Research, Physical Medicine and Rehabilitation, Feinberg School of Medicine, Northwestern University and Rehabilitation Institute of Chicago, Chicago, Illinois.

Supported by the National Institute on Disability and Rehabilitation Research through the Rehabilitation Research and Training Center on Substance Abuse, Disability, and Employment at Wright State University
(Grant No. H133B040012). The authors gratefully acknowledge the contributions of Susan Fraker and Sandy Castle of Wright State University and Scarlett Baker and Brandon Wires from the SASSI Institute. Muchappreciated feedback and encouragement was provided by the Illinois Department of Rehabilitation Services (Robert Kilbury, Director) and the Ohio Rehabilitation Services Commission (John Connelly, former Director).

Correspondence concerning this article should be addressed to Debra Guthmann, EdD, California School for the Deaf, 39350 Gallaudet Drive, Fremont, CA 94538. E-mail: Dguthmann@aol.com 
of the general population aged 12 or older was classified with substance dependence or abuse in the past year based on criteria specified in the Diagnostic and Statistical Manual of Mental Disorders, 4th edition (DSM-IV) (Substance Abuse and Mental Health Services Administration, 2010). There are at least one million functionally deaf persons in the United States (Mitchell, 2005), suggesting that at least 90,000 deaf persons in the United States may have a diagnosable SUD annually.

Deaf persons may experience an elevated risk of SUD for a number of reasons. Many deaf and hard-of-hearing persons have less access to educational information about alcohol and other drugs than their hearing peers. School prevention programs and media information often preclude access by deaf people due to a lack of captioned or signed materials, use of unfamiliar vocabulary, and other communication-related barriers. Historically, few residential schools for the deaf and almost no mainstream public school programs involve deaf and hard-of-hearing students in alcohol and drug abuse curricula (Berman, Steja, \& Guthmann, 2010; McCrone, 1982; Titus \& Guthmann, 2010). Increasingly, high schools require that students pass a high school exit examination. This requirement places pressure on schools serving deaf and hard-of-hearing students to focus on academic subjects, with one consequence being minimal emphasis on drug and alcohol and other pertinent life skills - related education. Additionally, there is a shortage of professionals working with deaf individuals (Pollard, 1996) and curricula focused on drug and alcohol education for persons who communicate in American Sign Language (ASL).

Family and social risk factors may also increase substance abuse risk among deaf individuals. Approximately $90 \%$ of all parents of deaf children are hearing (Mitchell, 2006; Mitchell \& Karchmer, 2004; Moores, 2001; Schein, 1974) and may not be able to communicate effectively with their children. Consequently, the process of "vertical enculturation" whereby parents transmit language, knowledge, and culture to their children is missing or delayed (Leigh \& Pollard, 2003). Communication among hearing and deaf family members is typically limited, and incidental learning through news reports and other media, as well as social discussions of local alcohol and drug use incidents and the dangers of substance use, is not always accessible to the deaf family members. The deaf child's desire to fit in with hearing peers and siblings, even those who use drugs, may influence his or her decision to use substances (Austen \& Chencinski, 2000; Dick, 1996; McCrone, 1982). Additionally, enabling may occur when hearing family members overlook classic symptoms of deaf family members' substance abuse and attribute them instead to hearing loss (Guthmann \& Graham, 2004).

For many deaf individuals, accessing spoken and written language is a struggle. While deaf people's written English may range from functional illiteracy to mastery (Brauer, Braden, Pollard, \& Hardy-Braz, 1998), the median reading comprehension for 17- and 18-year-old students is at the fourth grade (Gallaudet Research Institute, 1996). Compounding deaf persons' access to substance abuse information is the lack of expertise on the part of hearing professionals in both the language and the culture of deaf persons (Guthmann \& Graham, 2004; Guthmann \& Blozis, 2001; Guthmann \& Sandberg, 1998; Pollard, 1996). Deaf people are part of a linguistic minority whose primary language is ASL. Brauer et al. (1998) have pointed out that professional competence in psychology and deafness requires sign language fluency, as well as knowl- edge of deafness from many perspectives and expertise in mental health. ASL is a visual language that uses gestures, facial expression, body movements, and finger spelling for the letters of the individual words, and it is a recognized language with a distinct grammar, syntax, and vocabulary. As with any other language, ASL is shaped by the culture of the people who use it (Stokoe, 1980). Together, deaf persons' difficulties in accessing spoken and written language and lack of expertise in deaf sociocultural issues and ASL on the part of hearing mental health professionals and substance abuse prevention educators potentially increase deaf persons' risk for SUD.

Written screening tools are not the preferred method of identifying SUD within the deaf population. A study using the CAGE questionnaire and Alcohol Use Disorders Identification Test indicated that deaf individuals experience substantial difficulty understanding questions on these frequently used screening instruments, owing in part to the use of English-language idioms (Alexander, DiNitto, \& Tidblom, 2005). Moreover, the semantics of substance use and abuse are unfamiliar to many persons who are deaf (Guthmann \& Sandberg, 1998).

Using sign language interpreters to translate written screening instruments is not an ideal screening strategy. Even when interpreters are available to serve in this capacity and have expertise in ASL, often they are not trained in the specific terms used in alcohol or drug abuse assessment and treatment (Guthmann \& Graham, 2004). Variance in translation of specialized terms can invalidate assessments and screenings. Moreover, such procedures often take hours of client and staff time that press limited resources. Consequently, there is a need to develop and evaluate a brief standardized screening instrument in ASL (a) to identify who is likely to have an SUD and rule out those who do not, (b) to make an initial approximation of the type of problems and severity, and (c) to guide referral to further assessment and treatment.

In 2005, the Rehabilitation Research and Training Center on Substance Abuse, Disability, and Employment (RRTC) at Wright State University, in collaboration with the Rehabilitation Institute of Chicago and the SASSI Institute, initiated research to develop and field test a substance abuse screener for persons with disabilities who apply for employment assistance from state-based vocational rehabilitation (VR) programs. We theorized that a specialized screener could address readability, accessibility, prescription drug use, and employment-related consequences of substance use. The instrument was based on items selected from the Substance Abuse Subtle Screening Inventory-3 (SASSI-3; Lazowski, Miller, Boye, \& Miller, 1998). The capacity of the SASSI Institute to support the instrument's use through an established distribution infrastructure, automated scoring, and individual reports on screening results were additional considerations. The provisional 75 -item Substance Abuse in Vocational Rehabilitation-Screener (SAVR-S) was validated and shortened using data from approximately 1,000 VR consumers with disabilities (Heinemann, Lazowski, Moore, Miller, \& McAweeney, 2008). At the request of VR personnel, and supplemented by funding from the National Institute on Disability and Rehabilitation Research, a separate, ASL-based instrument was created from the SASSI-3 and new prescription drug use items (Guthmann \& Moore, 2007). 


\section{Study Objectives}

Our objectives were to develop and validate a substance use screening instrument in ASL to be used to identify deaf individuals who have a high probability of having an SUD so that they might be evaluated for treatment needs. Our goal was to develop an accurate screening instrument that balanced sensitivity and specificity while imposing minimal response burden on participants.

Modifications of the provisional version of the SAVR-S that was being developed for more general use for clients with disabilities were needed to accommodate the needs of deaf individuals due to readability, wording, regional differences in signing words or phrases, and instrument length.

\section{Method}

\section{Sample}

We recruited 198 deaf participants in five states (California, Minnesota, New Jersey, Ohio, and Washington) from 10 residential and outpatient behavioral health and family social service programs that provide specialized services for deaf individuals, as well as from two residential schools for the deaf.

The sample included persons who were White (47\%), African American (17\%), Asian (4\%), Native Hawaiian (3\%), Native American (3\%), and biracial or other races $(27 \%)$. Thirty percent were of Hispanic origin. Men composed $55 \%$ of the sample. Marital status included $66 \%$ never married, $13 \%$ part of an unmarried couple, $10 \%$ were divorced, $6 \%$ married, and $5 \%$ were separated or widowed. Education level included $26 \%$ with less than a high school degree, $70 \%$ had a high school degree or equivalent, $2 \%$ had less than 2 years of postsecondary education, and $2 \%$ had a postsecondary degree. Mean years of education was $11.8(S D=1.3$; range: $9-21)$. The mean age was 30.8 years $(S D=$ 13.4; range: $18-71$ ).

\section{Diagnostic Evaluations and Screening Instrument Items}

SUD diagnosis. Diagnostic evaluations of participants' current status on DSM criteria for SUD were provided by certified mental health or substance abuse counselors at programs that provide specialized services for deaf individuals. All counselors were either deaf or hearing, fluent in ASL, and conducted their evaluations in individual sessions with participants. Faculty from school settings were credentialed in the area of counseling, familiar with DSM nosology, and ASL-fluent.

Initial SAS-ASL item pool. The screening instrument was derived from the SASSI- 3 . The SASSI- 3 consists of 67 true-false items that include items with no apparent relationship to substance misuse as well as items to identify random answering; 12 items that ask clients to report experienced consequences of alcohol use; and 14 items pertaining to the abuse of illicit drugs. Based on recommendations of focus groups of counselors who work with clients with disabilities, items were added to the item pool for the SAS-ASL to assess misuse of prescription medications. The initial version of the SAS-ASL (Guthmann \& Moore, 2007) contained 75 items, including items to identify random answering and prescription drug abuse, as well as items used in the standard
SASSI-3 screening result regarding likelihood of SUD. These items were translated into ASL by a linguistics committee of deaf professionals who were native ASL users. Instrument length was reduced through extensive evaluations of back-translations provided by 37 deaf individuals from 19 states and multiple regions throughout the United States; ASL was their preferred mode of communication. Analyses of participants' back-translations and the linguistic committee's recommendations yielded 42 items (including 40 items to evaluate likelihood of SUD and two to identify random responding) that demonstrated consistent translations in both ASL and English; these items comprised the item pool for the validation phase of this research. Further details of the translation project and challenges encountered can be found in Guthmann and Moore (2007); see also Graybill et al. (2010).

\section{Rating Scale Analyses}

We used the Rasch rating scale model to evaluate the characteristics of the 40 substance use screening items on the SAS-ASL and to estimate the level of the people on the underlying dimension being measured-in this case, SUD. The Rasch measurement model is used frequently in developing and revising instruments used in education, quality of life, and health (Bezruczko, 2005; Rasch, 1980). Rasch analysis includes a family of models to calibrate items with different characteristics. Because the SAS-ASL uses ordinal rating scales, we used the Rasch rating scale model (Wright \& Stone, 1999). There are several criteria to assess the quality of an instrument using the Rasch model. Person separation values greater than 2.0 indicate the ability to distinguish at least three levels of the construct in the sample (Bond \& Fox, 2007). This value is equivalent to person separation reliabilities of .80 and is interpreted similarly to Cronbach's alpha. Fit to the Rasch model is interpreted as evidence of internal validity. Item fit to the Rasch model is evaluated with infit mean square statistics; values between 0.7 and 1.4 for individual items are optimal (Bond \& Fox, 2007). We used Winsteps, Version 3.68.0 (Linacre \& Wright, 2009).

\section{Results}

Of the 198 participants who were interviewed and completed the SAS-ASL screener, six produced elevated scores on the Random Answering Pattern items indicating that they had not responded to the questions on the screener in a meaningful manner. These six cases were excluded from further analyses. Diagnostic interview data indicated that $39.1 \%$ of the sample met DSM criteria for current SUD. Participants had been purposely recruited from substance use treatment programs and other behavioral health programs to assure that a substantial proportion of the sample would be criterion positive.

\section{Discriminant and Rating Scale Analyses}

Analyses were conducted to assess how well responses to each of the 40 SAS-ASL items related to substance misuse (i.e., excluding items used to identify random responding), discriminated between participants identified as having and as not having current SUD, and to evaluate the internal consistency of the items 
with the aim of producing a brief instrument that was accurate and minimized respondent burden.

In prior research with hearing participants, the written English equivalents of the items on the SAS-ASL had been shown to discriminate clients who did or did not have an SUD (Heinemann et al., 2008; Lazowski et al., 1998). In the current sample, seven items were not significant discriminators (s2, 6, 8, 11, 12, 13, and a8). These items included content such as, "feeling worn out for no reason," "knowing others with bad reputations," "feeling energized for days at a time without sleep," and "reexperiencing the effects of alcohol or drugs when sober."

We used rating scale analyses to assess the psychometric characteristics of the SAS-ASL 40-item set, including person reliability and item-measure correlations. Table 1 summarizes findings for five rating scale analyses: (a) the full 40 item set, (b) alcohol items, (c) drug items, (d) subtle items, and (e) a reduced set of 28 items. Findings indicated person reliability ranged from .89 (all 40 items) to .40 (alcohol items).

Rating scale analyses supported the discriminant analysis findings by indicating that each of the seven nondiscriminating items also correlated weakly $(<0.4)$ with the overall measure. In addition, two of these items (s2, "feeling worn out" and 13, "taking responsibilities seriously") as well as one item, which was significant in the discriminant function analysis (s17), had mean square infit values that exceeded the 1.4 threshold, indicating noisy fit to the underlying construct (Bond \& Fox, 2007). We decided to retain item 17 about family history of substance abuse because of its clinical utility, in addition to its ability to discriminate a somewhat different aspect of the SUD construct. Removing the item reduced sensitivity of the item set by $3 \%$. To decrease the total number of items on the instrument, we eliminated five additional items that were significant discriminators but showed low item-measure correlations or low weights in the discriminant function equation ( $\mathrm{s} 3,5,20$, a5, and $\mathrm{d} 3$ ) along with the seven items already noted, producing a final item set of 28 items. As shown in Table 1 , person reliability for the 28 -item set was .85 , exceeding the criterion of .80 .

Table 2 shows the item characteristics for the 28-item Rasch solution. By selection, only one item (s17) had a mean square infit value above 1.4, and this item was the only one that had a correlation of less than 0.4 with the total measure. Figure 1 is a map of item difficulties on the right and SAS-ASL measures for persons on the left. Consistent with the observed SUD prevalence of $39 \%$ in the sample, the majority of respondents reported little substance use and few problems, as illustrated by the frequency of persons below the easiest-to-endorse items. The item set was able to distinguish a range of substance use problems very well.

\section{Test-Retest Reliability}

We explored test-retest reliability by recruiting participants at a substance use treatment program that provides specialized services for deaf individuals, as well as students who were 18 years or older and attended a residential school for the deaf. These individuals were asked to complete the SAS-ASL twice within a 9- to 14-day interval. Twenty-seven participants viewed the SAS-ASL video, recorded their responses on the SAS-ASL paper questionnaire, and repeated this procedure approximately two weeks later. Pearson correlations on the instrument indicated stability coefficients of .96 for the subtle items, .93 for the alcohol items, and .89 for the drug items. All coefficients were statistically significant $(p<.01)$.

\section{Correspondence of SAS-ASL Identification With DSM Diagnoses}

We randomly selected one half of the cases in each of the diagnostic criterion groups (SUD, non-SUD) to serve as the development sample, which was used to develop a scoring routine for the instrument, and reserved the remaining cases to serve as the validation sample. Table 3 shows the correspondence between SAS-ASL screening classifications and clinical diagnostic classifications.

Using development sample responses and an iterative process of adjusting cutoff scores, we produced a scoring algorithm with $89 \%$ sensitivity, 68\% positive predictive value (PPV), $74 \%$ specificity, and $92 \%$ negative predictive value (NPV); likelihood ratio $(1, N=$ $94)=38.9, p<.001$. Applying the SAS-ASL scoring algorithm to the reserved validation sample yielded overall accuracy of $87 \%$, sensitivity of $90 \%$, PPV of $80 \%$, specificity of $84 \%$, and NPV of $92 \%$; likelihood ratio $(1, N=98)=59.6, p<.001$. Effect sizes observed in the correspondence between the SAS-ASL decision rule and the diagnostic classification of SUD, measured as $\Phi$, were 0.61 (Cohen's $d=1.5$ ) for the development sample and 0.74 (Cohen's $d=2.2$ ) for the cross-validation sample. These values represent large effect sizes (Cohen, 1988).

To test how well the validation findings generalized to an independent sample of deaf persons and to assess how long it took participants to complete the screener, the finalized SAS-ASL was recompiled without the eliminated items and administered to 65

Table 1

Summary of Substance Abuse Screener in American Sign Language Rating Scale Analyses (n = 192)

\begin{tabular}{lcccc}
\hline \multicolumn{1}{c}{ Item groups } & $\begin{array}{c}\text { Person } \\
\text { reliability }\end{array}$ & $\begin{array}{c}\text { Items with mean } \\
\text { square infit }>1.4\end{array}$ & Ceiling/floor effect & Item-measure $r$ s $<.40$ \\
\hline $\begin{array}{l}1.40 \text { items }(9 \text { alcohol, } \\
12 \text { drug, } 19 \text { subtle) }\end{array}$ & .89 & $\mathrm{~s} 2, \mathrm{~s} 13, \mathrm{~s} 17$ & None & $\mathrm{s} 2, \mathrm{~s} 3, \mathrm{~s} 5, \mathrm{~s} 6, \mathrm{~s} 8, \mathrm{~s} 11, \mathrm{~s} 12, \mathrm{~s} 13$, \\
$\begin{array}{l}\text { 2. } 9 \text { alcohol items } \\
\text { 3. } 12 \text { drug items }\end{array}$ & .40 & None & $3 \%$ at ceiling, $42 \%$ at floor & None \\
4. 19 subtle items & .70 & $\mathrm{~d} 12$ & $4 \%$ at ceiling, $42 \%$ at floor & None \\
5. 28 items $(7$ alcohol, & .63 & $\mathrm{~s} 17$ & $5 \%$ at ceiling, $16 \%$ at floor & None \\
11 drug, 10 subtle) & .85 & $\mathrm{~s} 17$ & $12 \%$ at floor & $\mathrm{S} 17$ \\
\hline
\end{tabular}

Note. Rating scale categories were yes or no for all items. 
Table 2

Substance Abuse Screener in American Sign Language 28-Item Version Rating Scale Statistics in "Measure" Order

\begin{tabular}{|c|c|c|c|c|}
\hline Item abbreviated item label & Measure $^{\mathrm{a}}$ & SEM & Infit $^{\mathrm{b}}$ & Correlation $^{c}$ \\
\hline d11. Used other people's medications & 1.55 & 0.25 & 0.89 & .48 \\
\hline d10. Higher medication dose than prescription & 1.04 & 0.22 & 1.06 & .45 \\
\hline a3. Drank to boost energy & 0.69 & 0.21 & 1.11 & .46 \\
\hline a4. Drank more than intended & 0.64 & 0.21 & 1.12 & .46 \\
\hline d8. Polydrug abuse & 0.53 & 0.20 & 0.74 & .62 \\
\hline a1. Drinks with lunch & 0.52 & 0.20 & 0.94 & .53 \\
\hline d1. Misused to improve mood & 0.52 & 0.20 & 0.90 & .56 \\
\hline d9. Misuse limited goals & 0.25 & 0.20 & 0.86 & .59 \\
\hline d7. Time in drug-related activity & 0.22 & 0.19 & 0.86 & .59 \\
\hline s15. Drink to steady nerves & 0.13 & 0.19 & 1.10 & .51 \\
\hline a9. Relationship problems & 0.13 & 0.19 & 1.10 & .50 \\
\hline s21. Binge use & 0.11 & 0.19 & 1.10 & .50 \\
\hline d5. Misused to forget pressures & 0.11 & 0.19 & 0.87 & .59 \\
\hline a2. Drank to express feelings & 0.09 & 0.19 & 1.19 & .46 \\
\hline d2. Misused to feel better & 0.03 & 0.19 & 0.85 & .60 \\
\hline d4. Misused to forget feelings & 0.02 & 0.19 & 0.87 & .60 \\
\hline a7. Argued with family, friends & -0.03 & 0.19 & 0.94 & .58 \\
\hline s10. Smoke cigarettes regularly & -0.08 & 0.19 & 1.00 & .54 \\
\hline d6. Got really stoned & -0.19 & 0.19 & 0.92 & .59 \\
\hline a6. Became depressed when sober & -0.20 & 0.19 & 1.17 & .49 \\
\hline d12. Accepted into treatment & -0.43 & 0.18 & 0.98 & .59 \\
\hline s14. Neglected obligations & -0.55 & 0.18 & 0.83 & .65 \\
\hline s18. Use leads to trouble & -0.73 & 0.18 & 1.00 & .60 \\
\hline s16. Began regular use as teen & -0.76 & 0.18 & 1.09 & .55 \\
\hline s7. Used too much alcohol, pot & -0.79 & 0.18 & 0.84 & .66 \\
\hline s4. Sometimes drink too much & -0.82 & 0.18 & 1.14 & .54 \\
\hline s17. Father heavy drinker/drug user & -0.95 & 0.18 & 1.64 & .29 \\
\hline s19. Use causes family problems & -1.05 & 0.18 & 0.97 & .62 \\
\hline$M$ & 0.00 & 0.19 & 1.00 & \\
\hline$S D$ & 0.61 & 0.01 & 0.17 & \\
\hline
\end{tabular}

Note. Items are arranged in descending order of difficulty of endorsement (see Measure column).

a Item difficulty in logits; item difficulties are anchored at a mean of 0 and $S D$ of $1 . \quad{ }^{\text {b }}$ Mean square infit statistic with expectation of 1 . Values greater than 1.4 indicate unexpected noise; values less than 0.7 indicate dependency in the data. ${ }^{\mathrm{c}}$ Correlation between item and measure.

respondents in clinical and educational settings that provide services to deaf individuals in California and Washington State. Following evaluations by counselors of each of the participants about their status on criteria for current SUDs, participants viewed the ASL video and recorded their responses. Three cases had elevated random answering scores and were excluded from further analyses. Sample participants were White (48\%), African American (7\%), Asian (10\%), Native Hawaiian (2\%), Native American $(3 \%)$, and biracial $(3 \%)$ or other races $(28 \%)$. Thirty-three percent were of Hispanic origin. Men composed $67 \%$ of the sample. Marital status included $69 \%$ never married, $10 \%$ part of an unmarried couple, $10 \%$ were divorced, $8 \%$ married, and $3 \%$ were separated or widowed. Education level included $13 \%$ with less than a high school degree, $55 \%$ had a high school degree or equivalent, $27 \%$ had less than 2 years of postsecondary education, and $5 \%$ had a postsecondary degree. Mean years of education was $12.3(S D=1.0$; range: $11-16)$. The mean age was 32.4 years $(S D=12.6$; range: $18-64)$.

It took participants an average of $15.9 \min (S D=6.6$; range: $5-28)$ to view the items on the video and to record their responses on the paper form. Although participants could record their yes-no responses to each ASL video item on the paper form simply by locating the item number on the form, we assessed the reading comprehension required to read the full English text of the items, should participants choose to do so. Findings for the written version of the SAS-ASL questionnaire indicated a Flesch-Kincaid Grade Level readability score of 4.3 with a Flesch Reading Ease score of 80.2.

Applying the SAS-ASL scoring system to participants' responses yielded $100 \%$ accuracy for all four validity indices; likelihood ratio (1, $N=62)=74.7, p<.001$, as shown in Table 3. The effect size for the correspondence between the SAS-ASL decision rule and the diagnostic classification of SUD, measured as $\Phi$, was 1.00 (Cohen's $d>2.0$ ), representing a large effect size (Cohen, 1988).

\section{Discussion}

The SAS-ASL instrument demonstrates strong psychometric properties especially in light of semantic challenges involved with ASL. The screening data demonstrate sensitivity (.90), specificity (.84), and person reliability (.85) that are impressive given the relatively small sample. These psychometric properties were replicated in an independent sample of 62 deaf persons and rival rigorous screeners in the hearing population. The average administration time of $16 \mathrm{~min}$ is an advantage when considering the longer interaction times typically necessary for communicating clinical information via ASL. It typically would take a counselor longer to evaluate a deaf person for 


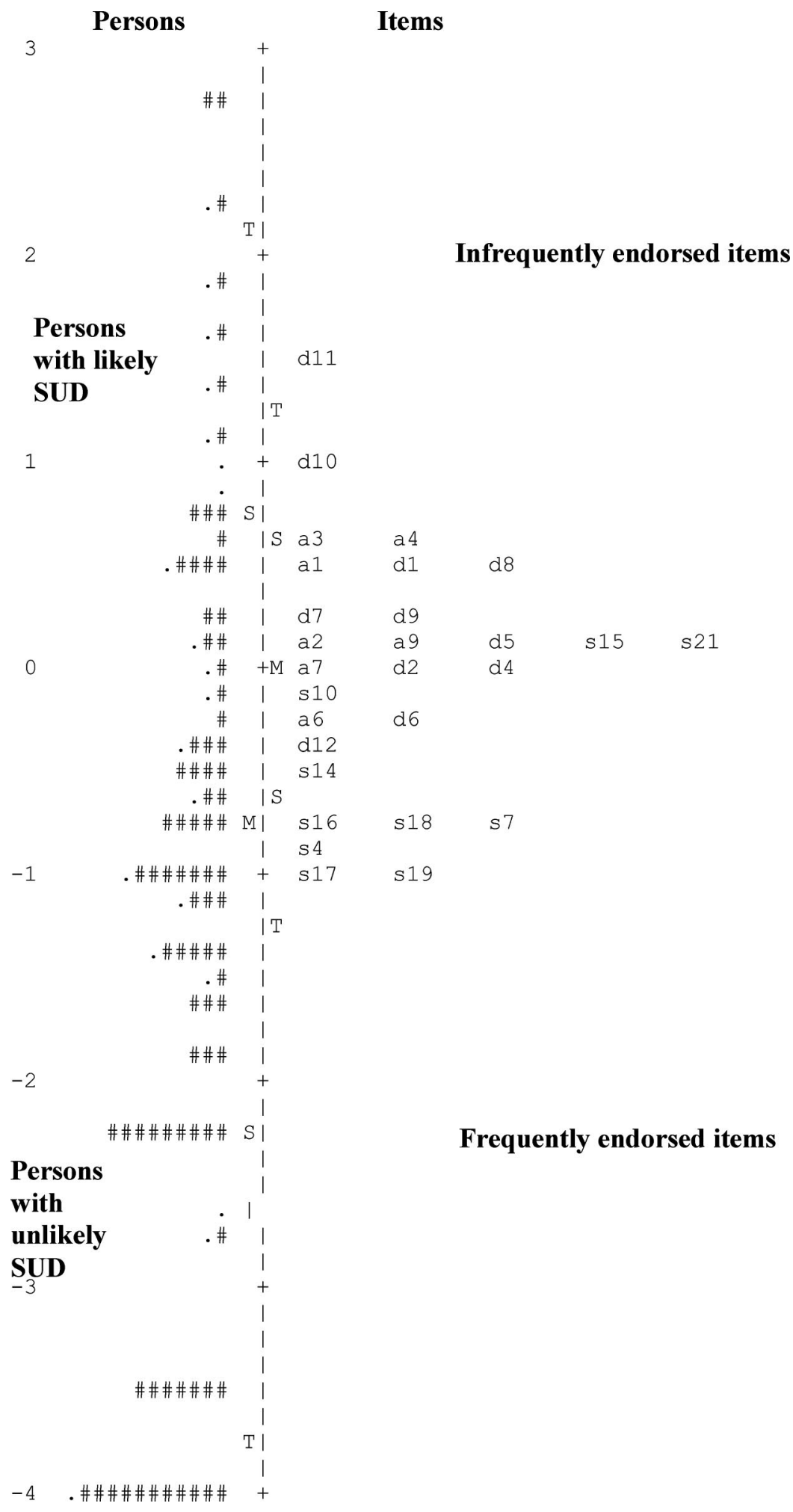

Figure 1. Map of items and persons on the 28-item SAS-ASL $(N=192)$. Each \# represents 2 respondents. The distribution of client measures (in log-odds units) is shown in the left histogram; the distribution of item difficulties is illustrated in the right histogram. Item labels correspond to items listed in Table 2. $M=$ mean; $S=$ 1 standard deviation. The large number of \# symbols at the bottom of the figure illustrates the skew in the sample toward low levels of SUD likelihood. 
Table 3

Correspondence of the 28-Item Substance Abuse Screener in American Sign Language (SAS-ASL) With DSM Diagnoses of Substance Use Disorders

\begin{tabular}{cccc}
\hline & \multicolumn{2}{c}{ SAS-ASL classification } & \\
\cline { 2 - 3 } $\begin{array}{c}\text { DSM diagnostic } \\
\text { classification }\end{array}$ & Test positive & Test negative & Total \\
\hline $\begin{array}{c}\text { Development sample } \\
\text { Criterion positive }\end{array}$ & 32 & 4 & \\
Criterion negative & $89 \%$ & 43 & 56 \\
Total & 15 & $74 \%$ & 58 \\
Validation sample & 47 & 47 & 94 \\
Criterion positive & 37 & 4 & 41 \\
Criterion negative & $90 \%$ & 48 & 57 \\
Total & 9 & $84 \%$ & \\
Second validation sample & & 52 & 98 \\
Criterion positive & 46 & 0 & 44 \\
Criterion negative & $100 \%$ & 18 & 18 \\
Total & 0 & $18 \%$ & 62 \\
\hline
\end{tabular}

Note. $\quad$ DSM $=$ Diagnostic and Statistical Manual of Mental Disorders.

${ }^{\mathrm{a}}$ Sensitivity $=89 \%$; specificity $=74 \%$; positive predictive value $(\mathrm{PPV})=$ $68 \%$; negative predictive value $(\mathrm{NPV})=92 \%$. ${ }^{\mathrm{b}}$ Sensitivity $=90 \%$; specificity $=84 \% ; \mathrm{PPV}=80 \% ; \mathrm{NPV}=92 \% . \quad{ }^{\mathrm{c}}$ Sensitivity $=100 \%$; specificity $=100 \% ; \mathrm{PPV}=100 \% ; \mathrm{NPV}=100 \%$.

SUD than it would for clients to complete the instrument; using the screener can help allocate limited resources.

An additional strength of the SAS-ASL is that it underwent extensive translation development to select comprehensible signing conventions that are functional across geographic regions of the United States. The methods employed in the translation/backtranslation of the instrument represent the current state of the art with regard to semantic and linguistic instrument development, because the process involved a team of ASL-fluent professionals and back translators from multiple regions of the United States who were culturally deaf, children of deaf adults, credentialed professionals in the field, or certified sign language interpreters. The resulting instrument is a standardized screening measure for accurate identification of likely SUD that can be used to facilitate screening and treatment referral in programs throughout the United States.

The availability of the SAS-ASL in electronic form, with the advantages of automated scoring and individual report generation, enhance access to substance abuse screening within an underserved population. Individualized content on SAS-ASL screening reports, including: loss of control in usage, experienced negative consequences, neglect of obligations due to use, substance use to manage emotions and cope with negative feelings, misuse of medications, physical tolerance and/or withdrawal, and prior treatment for SUD can aid counselors in treatment planning and referral. Those interested in the SAS-ASL may contact coauthor Debra Guthmann, the SASSI Institute, or go to http:// www.sassi.com/srs/sas-asl.html for further information.

The evolving availability of behavioral health care that utilizes e-therapy increases the potential utility and applicability of this screening tool for persons and agencies with limited deaf-specific resources. A semantically and clinically accurate screener is the first step in providing assistance to persons who communicate primarily through sign language. It is not well understood by the larger behavioral health provider communities, but the availability of a trained sign language interpreter is a less than ideal alternative for clinical interaction with persons who are deaf. There are multiple issues that attenuate the quality of care provided in the triad of clinician, interpreter, and client (Guthmann \& Graham, 2004; Titus, Schiller, \& Guthmann, 2008). These concerns can be eliminated when conducting behavioral health screenings with the SAS-ASL. The use of the SAS-ASL could constitute the first step in a system of triage and treatment that combines the screener with e-therapy or web-based support groups. Systems that use local treatment resources and online deaf-specific therapies have been shown to have particular promise for this low-incidence population (Moore, Guthmann, Rogers, Fraker, \& Embree, 2009). Because it is challenging to provide culturally sensitive and trained mental health professionals fluent in ASL in all but the larger metropolitan areas, the use of technology can extend this expertise to other geographic areas. For example, the program administered through Wright State University has established a network whereby anonymous self-help support groups in ASL can be web-based and operate daily, be led by deaf individuals in recovery, and involve deaf group members from as far away as Europe or Asia (Moore et al., 2009).

There is a history of research on the extended risk factors for SUD by persons with disabilities, and persons who are deaf may represent the subgroup most at risk due to cultural, communication, and structural limitations (Guthmann \& Moore, 2007; Moore \& Polsgrove, 1991; Substance Abuse and Mental Health Services Administration, 1998). Despite continuing advances in civil rights for persons with disabilities, as well as positive changes actualized through technology, persons who are deaf continue to be at risk for societal disenfranchisement. Multiple factors contribute to an unacceptably high rate of behavioral disorders among persons in this population (Moore \& McAweeney, 2006/2007). One of the first steps in addressing these conditions is to accurately identify persons at risk for behavioral problems. The development and validation of the SAS-ASL brings appropriate behavioral health care one step closer to reality for persons who are deaf.

\section{Study Limitations}

The instrument's limitations should be noted. The SAS-ASL is intended for screening purposes only-it does not provide a diagnosis. Despite the provision of back-translation data from almost 40 deaf individuals from numerous regions throughout the United States, some may not be familiar with the signs selected to convey alcohol, illicit drug, and prescription drug use and misuse terminology. It is also possible that inclusion of written English item content on the answer forms could prove distracting to participants who do not have at least a fourth grade level of English reading comprehension. We valued the brevity of the screening instrument and in so doing eliminated items designed to detect response minimization; therefore, it is possible the screener will miss individuals who attempt to conceal their use. Finally, the screening accuracy rates observed in these samples from behavioral health and family social service programs that provide services to deaf 
persons may not be representative of all such programs and their replication awaits further investigation.

\section{Conclusion}

We developed a 28-item instrument in ASL to screen for SUDs in deaf individuals. The SAS-ASL demonstrates high sensitivity and good specificity; it requires approximately $16 \mathrm{~min}$ to complete. The instrument can be used to provide access to standardized, culturally appropriate, and psychometrically sound SUD screening for deaf individuals and can be self-administered, which may be particularly valuable in programs where deaf-specific resources are limited. The software platform developed to support the instrument's use provides counselor access to the SAS-ASL and support documents via the Internet, as well as automated scoring and individualized reports on screening results via email or fax. The SAS - ASL can be used to assist counselors in identifying deaf persons in need of further evaluation for SUD and facilitate treatment planning based on individualized SUD content areas identified on the screening reports.

\section{References}

Alexander, T., DiNitto, D., \& Tidblom, I. (2005). Screening for alcohol and other drug problems among the deaf. Alcoholism Treatment Quarterly, 23, 63-78. doi:10.1300/J020v23n01_06

Austen, S., \& Checinski, K. (2000). Addictive behavior and deafness. In P. Hindley \& N. Kitson (Eds.), Mental health and deafness (pp. 232-252). Philadelphia, PA: Whurr Publishers.

Berman, B. A., Steja, L., \& Guthmann, D. S. (2010). Alcohol and other substance use among deaf and hard of hearing youth. Journal of Drug Education, 40(2), 99-124.

Bezruczko, N. (2005). Rasch measurement in health sciences. Maple Grove, MN: JAM Press.

Bond, T. G., \& Fox, C. M. (2007). Applying the Rasch model: Fundamental measurement in the human sciences (2nd ed.). Mahwah, NJ: Lawrence Erlbaum.

Brauer, B. A., Braden, J. P., Pollard, R. Q., \& Hardy-Braz, S. T. (1998). Deaf and hard-of-hearing people. In J. Sandoval, C. L. Frisby, K. F. Geisinger, J. Dowd Scheuneman, \& J. Ramos Grenier (Eds.), Test interpretation and diversity: Achieving equity in assessment (pp. 297315). Washington, DC: American Psychological Association. doi: 10.1037/10279-011

Cohen, J. (1988). Statistical power analysis for the behavioral sciences (2nd ed.). Hillsdale, NJ: Erlbaum.

Dick, J. (1996). Signing for a high: A study of alcohol and drug use by deaf and hard of hearing adolescents. Dissertation Abstracts International, 57(6A), 2675.

Gallaudet Research Institute. (1996). Stanford Achievement Test, 9th edition, Form S, Norms booklet for deaf and hard-of-hearing students. Washington, DC: Gallaudet University.

Gallaudet Research Institute. (2005). Regional and national summary report of data from the 2003-2004 annual survey of deaf and hard of hearing children and youth. Washington, DC: GRI, Gallaudet University.

Graybill, P., Aggas, J., Dean, R. K., Demers, S., Finigan, E., \& Pollard, R. Q. (2010). A community-participatory approach to adapting survey items for deaf individuals and American Sign Language. Field Methods, 22, 429-448. doi:10.1177/1525822X10379201

Guthmann, D., \& Blozis, S. A. (2001). Unique issues faced by deaf individuals entering substance abuse treatment and following discharge. American Annals of the Deaf, 146(3), 294-304

Guthmann, D., \& Graham, V. (2004). Substance abuse: A hidden problem within the D/deaf and hard of hearing communities. Journal of Teaching in the Addictions, 3, 49-64. doi:10.1300/J188v03n01_04

Guthmann, D., \& Moore, D. (2007). The Substance Abuse in Vocational Rehabilitation Screener in American Sign Language (SAVR-S-ASL) for persons who are deaf. Journal of the American Deafness and Rehabilitation Association, 41(1), 9-16.

Guthmann, D., \& Sandberg, K. (1998). Assessing substance abuse problems in deaf and hard of hearing individuals. American Annals of the Deaf, 143(1), 14-21.

Heinemann, A. W., Lazowski, L. E., Moore, D., Miller, F., \& McAweeney, M. (2008). Validation of a substance use disorder screening instrument for use in vocational rehabilitation settings. Rehabilitation Psychology, 53, 63-72. doi:10.1037/0090-5550.53.1.63

Lazowski, L. E., Miller, F. G., Boye, M. W., \& Miller, G. A. (1998). Efficacy of the Substance Abuse Subtle Screening Inventory-3 (SASSI-3) in identifying substance dependence disorders in clinical settings. Journal of Personality Assessment, 71, 114-128. doi:10.1207/ s15327752jpa7101_8

Leigh, I. W., \& Pollard, R. Q. (2003). Mental health and deaf adults. In M Marschark \& P. E. Spencer (Eds.). Oxford handbook of deaf studies, language, and education (pp. 203-215). New York, NY: Oxford University Press.

Linacre, J. M., \& Wright, B. D. (2009). Winsteps (Version 3.68.0). Chicago, IL: Winsteps.

Lipton, D., \& Goldstein, M. (1997). Measuring substance abuse among the deaf. Journal of Drug Issues, 27, 733-754.

McCrone, W. P. (1982). Serving the deaf substance abuser. Journal of Psychoactive Drugs, 14, 199-203. doi:10.1080/02791072.1982 .10471929

Mitchell, R. E. (2006). How many deaf people are there in the United States? Estimates from the Survey of Income and Program Participation. Journal of Deaf Studies and Deaf Education, 11, 112-119.

Mitchell, R. E., \& Karchmer, M. A. (2004). Chasing the mythical ten percent: Parental hearing status of deaf and hard of hearing students in the United States. Sign Language Studies, 4, 138-163. doi:10.1353/ sls. 2004.0005

Moore, D., Guthmann, D., Rogers, N., Fraker, S., \& Embree, J. (2009). E-therapy as a means for addressing barriers to substance use disorder treatment for persons who are deaf. Journal of Sociology and Social Welfare, 36(4), 75-92.

Moore, D., \& McAweeney, M. J. (2006/2007). Demographic characteristics and rates of progress of deaf and hard of hearing persons receiving substance abuse treatment. American Annals of the Deaf, 151, 508-512. doi:10.1353/aad.2007.0005

Moore, D., \& Polsgrove, L. (1991). Disabilities, developmental handicaps, and substance misuse: A review. International Journal of the Addictions, 26(1), 65-90.

Moores, D. F. (2001). Educating the deaf: Psychology, principles, and practices. Boston, MA: Houghton Mifflin.

Pollard, R. Q. (1996). Professional psychology and deaf people: The emergence of a discipline. American Psychologist, 51, 389-396. doi: 10.1037/0003-066X.51.4.389

Rasch, G. (1960/1980). Probabilistic models for some intelligence and attainment tests. (Copenhagen, Danish Institute for Educational Research), expanded edition (1980) with foreword and afterword by B.D. Wright. Chicago, IL: The University of Chicago Press.

Schein, J. (1974). The deaf population of the United States. Silver Springs, MD: National Association of the Deaf.

Stokoe, W. C. (Ed). (1980). Sign and culture: A reader for students of American Sign Language. Silver Springs, MD: Linstok Press.

Substance Abuse and Mental Health Services Administration. (1998). Substance use disorder treatment for people with physical and cognitive disabilities: Treatment Improvement Protocol (TIP) (Series 29, No. SMA 98-3249). Washington, DC: Author. 
Substance Abuse and Mental Health Services Administration. (2010). Results from the 2009 National Survey on Drug Use and Health: Volume I. Summary of national findings (NSDUH Series H-38A, No. SMA 10-4856 Findings). Rockville, MD: Author.

Titus, J., \& Guthmann, D. (2010). Addressing the black hole in substance abuse treatment for deaf and hard of hearing individuals: Technology to the rescue. Journal of the American Deafness and Rehabilitation Association, 43(2), 92-100.

Titus, J. C., Schiller, J. A., \& Guthmann, D. (2008). Characteristics of youths with hearing loss admitted to substance abuse treatment. Journal of Deaf Studies and Deaf Education, 13, 336-350. doi:10.1093/deafed/ enm068

Wright, B. D., \& Stone, M. H. (1999). Measurement essentials (2nd ed.). Wilmington, DE: Wide Range.

Received May 10, 2011

Revision received April 11, 2012

Accepted April 16, 2012

\section{Members of Underrepresented Groups: Reviewers for Journal Manuscripts Wanted}

If you are interested in reviewing manuscripts for APA journals, the APA Publications and Communications Board would like to invite your participation. Manuscript reviewers are vital to the publications process. As a reviewer, you would gain valuable experience in publishing. The P\&C Board is particularly interested in encouraging members of underrepresented groups to participate more in this process.

If you are interested in reviewing manuscripts, please write APA Journals at Reviewers@apa.org. Please note the following important points:

- To be selected as a reviewer, you must have published articles in peer-reviewed journals. The experience of publishing provides a reviewer with the basis for preparing a thorough, objective review.

- To be selected, it is critical to be a regular reader of the five to six empirical journals that are most central to the area or journal for which you would like to review. Current knowledge of recently published research provides a reviewer with the knowledge base to evaluate a new submission within the context of existing research.

- To select the appropriate reviewers for each manuscript, the editor needs detailed information. Please include with your letter your vita. In the letter, please identify which APA journal(s) you are interested in, and describe your area of expertise. Be as specific as possible. For example, "social psychology" is not sufficient-you would need to specify "social cognition" or "attitude change" as well.

- Reviewing a manuscript takes time (1-4 hours per manuscript reviewed). If you are selected to review a manuscript, be prepared to invest the necessary time to evaluate the manuscript thoroughly. 